\title{
Psychiatric Factors and Weight Loss Patterns Following Gastric Bypass Surgery in a Veteran Population
}

\author{
Thomas Rutledge • Lisa M. Groesz • Michelle Savu
}

Received: 10 April 2009 / Accepted: 30 June 2009/Published online: 15 July 2009

(C) The Author(s) 2009. This article is published with open access at Springerlink.com

\begin{abstract}
Background Although pre-surgical psychological evaluations are commonly administered to patients considered for weight loss surgeries, the value of these evaluations for predicting weight loss success has been questioned. In this study, we addressed this issue by examining patient's total number of psychiatric indicators rather than individual psychological factors as predictors of weight loss/weight regain.

Methods Sixty adult veterans completed gastric bypass surgery or laparoscopic gastric banding after completing a multidisciplinary evaluation for surgical clearance, including a psychological assessment. Patients were subsequently followed for 24 month to examine changes in weight, body mass index, and risk of weight regain.

Results Nearly three fourths of the sample carried either a single (40.0\%) or multiple psychiatric diagnoses (33.4\%). Nearly half $(47.5 \%)$ of the sample ceased losing weight
\end{abstract}

T. Rutledge $\cdot$ M. Savu

VA San Diego Healthcare System,

San Diego, CA, USA

T. Rutledge

University of California, San Diego,

San Diego, CA, USA

\section{M. Groesz}

University of California, San Francisco,

San Francisco, CA, USA

T. Rutledge $(\bowtie)$

Psychology Service 116B, VA San Diego Healthcare System,

Medical Center,

3350 La Jolla Village Drive,

San Diego, CA 92161, USA

e-mail: Thomas.Rutledge@va.gov after 1 year, with $29.5 \%$ regaining weight after this point. Neither psychiatric nor biomedical factors effectively predicted 1-year weight loss totals; however, we observed a significant linear relationship between the number of psychiatric factors and post-1-year weight changes $(r=0.33$, $p=0.01$ ). After adjusting for demographic and biomedical factors, patients with two or more psychiatric diagnoses were found to be significantly more likely to experience weight loss cessation or weight regain after 1-year $(\mathrm{OR}=$ $6.4,95 \% \mathrm{CI}=1.3-12.4)$ relative to those with zero or one psychiatric diagnosis.

Conclusions Assessing mental health factors in terms of the total number of psychiatric conditions improved the prediction of weight loss surgery outcomes in this veteran sample. An expanded model for understanding how psychological factors may affect weight loss surgery may improve the utility of pre-bariatric psychological assessments.

Keywords Weight loss surgery · Psychological · Veteran

Weight loss surgery is an increasingly common treatment for obesity $[1,2]$. In the evaluation of candidates for weight loss surgery, a pre-surgical psychological assessment is a standard component of the appraisal process [3, 4]. A survey of 81 bariatric surgery centers in the USA [4] indicated that nearly $90 \%$ required some form of psychological evaluation prior to weight loss surgery. The prevalence of psychiatric disorders in bariatric surgery candidates appears high based on the best available diagnostic methods. For example, Kalarchian and colleagues [5] used standardized clinical interviews for the assessment of psychiatric disorders in 288 pre-bariatric surgery patients, observing that nearly two thirds met criteria for a lifetime axis I disorder and 38\% met criteria for an axis I disorder at the time of surgery. In practice, the 
results of psychological assessments are considered in combination with factors such as the patient's age, medical status, and social circumstances in evaluating the riskbenefit ratio of proceeding with surgery [6-8].

Given the near universal consensus for the need of presurgical psychological evaluations, it is surprising that psychosocial factors are often weak predictors of weight loss surgery outcomes in published research. Separate literature reviews led by Van Hout et al. [9], Herpertz et al. [10], Greenberg et al. [11], Bocchieri et al. [12], and Sarwer et al. [13], for example, each described poor consistency across studies regarding psychological factors as predictors of variation in surgical weight loss and even a lack of consensus regarding which factors may be most important. In a recent study of morbidly obese patients undergoing vertical-banded gastroplasty [14], in which a battery of psychosocial measures failed to predict 2-year weight changes, the authors even suggested that "preoperative psychological assessment may not be necessary with respect to weight loss." The routinely observed result that weight loss surgery improves patient's quality of life and mental health - often irrespective of background factors such as psychiatric disorders, binge eating, or emotional eating histories - could be seen as further support for the sentiment that psychiatric status is not critical to weight loss surgery outcomes $[12,15,16]$.

An alternative perspective explored in some recent studies is that psychological factors may become more important to weight loss surgery outcomes when they are studied in combination. For example, Kinzl et al. [17] described associations between psychiatric disorders and weight loss following laparoscopic adjustable gastric banding, finding that psychiatric disorders were associated with significantly lower weight loss over a median period of 50-month follow-up but only among patients with two or more psychiatric diagnoses. The latter result suggests that it is the overall burden or severity of psychological factors rather than the simple presence of a psychiatric diagnosis that may place the patient at greater risk of poor weight loss surgery outcomes.

Studies addressing relationships between psychological factors and weight loss surgery outcomes may also benefit from expanding the definition of poor surgery outcomes beyond weight loss. For example, rates of weight regain following surgery may be a more effective long-term indicator of weight loss surgery success. Based on results reported by Christou et al. [18], substantial weight regain may undermine the results of one fifth to one third of weight loss surgery patients. Similar rates of weight regain have been observed in long-term follow-up from the Swedish Obesity Study [19]. Although some amount of weight gain can be considered normal in weight loss surgery patients followed long term, weight regain or the absence of continued weight loss in the in the initial months following surgery may be especially concerning.

In the current study of veteran patients completing gastric bypass surgery or laparoscopic adjustable gastric banding, we assessed the relationship between patient's total number of psychiatric conditions and weight loss surgery outcomes in the form of 1-year weight loss and rates of weight regain over 24 months of follow-up. We hypothesized that a greater number of psychiatric conditions would be associated with poorer initial weight loss and higher rates of weight regain.

\section{Method}

\section{Participants}

The study sample comprised 60 adult veterans completing weight loss surgery (laparoscopic adjustable gastric banding and gastric bypass surgery) through the weight control clinic of the VA San Diego Healthcare System between 1998 and 2007. Participants were approved for surgery after a multidisciplinary evaluation by a staff physician, psychologist, and weight loss surgeon, among other disciplines as deemed appropriate. Participants had a minimum body mass index $(\mathrm{BMI}) \geq 35$, age $\leq 65$, spoke English as their primary language, had no history of substance abuse or psychiatric admissions in the past 12 months or a previous diagnosis of dementia, and were preoperatively evaluated and medically cleared by staff physicians (endocrinologist or internal medicine). As routine part of obtaining clearance for surgery at the participating institute, participants achieved a minimum 5\% weight loss through non-surgical methods prior to surgery.

The study methods included both retrospective and prospective components, with participants tracked after surgery through the computerized medical record system in the VA. Retrospective data obtained included a review of participant's medical histories, results of pre-surgical evaluations, medical and psychiatric diagnoses, and active medication prescriptions at the time of weight loss surgery. Participants were subsequently followed for care through the weight control clinic and other VA hospital services following their surgery. University and VA Institutional Review Board approval was obtained as part of the study completion.

\section{Biomedical Factors}

For the determination of medical status, the participant's history and physical (H\&P) exam completed for their surgical admission were used to identify current medical diagnoses, medication use, and medical histories. For all 
participants, the H\&P exam was performed within 2 weeks prior to their admission for surgery and updated the day of surgery. Participant's medical records in the year prior to surgery were also assessed for visits to specialty clinics for surgeries, medications, and other treatments supporting a disease diagnosis. Using these methods, we specifically assessed for the presence of cardiovascular disease risk factors (hypertension, diabetes, dyslipidemia, and smoking) and related conditions including sleep apnea and erectile/ sexual dysfunction. BMI and weight were tracked at surgery admission, 6 months, 1 year, and 2 years.

\section{Mental Health and Demographic Assessment}

Information concerning participant's mental health status was drawn from several sources. These sources included the pre-surgical evaluation with a licensed psychologist, the physician H\&P visit, mental health visits in the year prior to surgery, and a review of active medications as of the time of surgery. From these sources, we coded psychiatric diagnoses including depression, anxiety disorders, binge eating or other eating disorders, and prior suicide attempts. Although suicide attempts do not represent a psychiatric disorder, they are one of the specific psychiatric factors most commonly used to identify high-risk weight loss surgery candidates [4]. Suicide risk is likewise a particularly important concern for male veterans, who have been shown to die from suicide at twice the rate of their civilian counterparts [20]. Demographic factors included age at date of surgery, gender, and racial background.

\section{Statistical Analyses}

Descriptive statistics, $t$ tests of independent groups, and chisquare analyses were used initially to examine demographic, biomedical, and psychiatric factors, including possible differences between patients completing gastric bypass versus laparoscopic adjustable gastric-banding procedures. Psychiatric status was calculated by summing the presence of dichotomous (yes/no) psychiatric conditions including depression, anxiety, binge eating, and a history of suicide attempts, with a score range on this variable from 0 to 4 . Because of the skewed form of the psychological burden variables (few participants had more than three conditions), we aggregated this variable into a 0,1 , and " 2 or more" categorical variable for final analyses. Outcomes consisted of short-term results measured by 1-year BMI and weight changes and long-term results defined by weight loss patterns at 24 months. Weight loss over 1 year following surgery was calculated using the history and physical exam admission weight recorded for the patient before surgery and weight recorded 1 year later in the computer record system to the nearest day. Between 1 and 2-year post- surgery dates, we further assessed the failure to either lose additional weight after or actual regain of weight. Weight loss cessation and weight regain were computed as both a continuous and dichotomous variables, calculated as the difference between participant's 1-year post-surgery weight and their 24-month post-surgery as documented in the hospital computerized record system. Relationships between psychiatric factors and long-term weight status/risk of weight loss cessation/weight regain were assessed using correlation coefficients and hierarchical regression in which we adjusted for demographic factors and pre-surgery BMI. The criterion for statistical significance was set at $p<0.05$. All calculations were performed using SPSS 17.0 (http:// www.SPSS.com).

\section{Results}

Demographic, medical, and psychiatric descriptors of the 60 veterans completing weight loss surgery are presented in Table 1 . The results are presented separately by surgical procedure. For the complete sample, patients experienced a mean 26.3\% [80.9 (41.9) lbs] weight loss after 1 year. Patients completing gastric bypass surgery were heavier prior to surgery compared to laparoscopic adjustable gastric-banding patients [BMIs=46.0 (7.5) and 39.5 (3.9) for the gastric bypass and laparoscopic adjustable gastric banding groups, respectively, $t(58)=2.9, p=0.005]$ and lost a larger percent of body weight in the initial 12 months following surgery [29.3\% versus $11.1 \%$ weight loss, respectively, $t(58)=5.6$, $p<0.001]$. Just four of 12 patients in the sample receiving the laparoscopic adjustable gastric banding procedure experienced 12-month weight loss $\geq 10 \%$, suggesting modest shortterm changes. However, the groups were otherwise similar in regards to pre-surgery cardiovascular risk factors and psychiatric conditions, both of which were present at moderate to high rates in this veteran population.

\section{Psychiatric Health and Patterns of Weight Loss} Following Surgery

Although psychiatric conditions such as depression and anxiety were common on their own, mental health factors tended to cluster in this sample. Among the participating sample, $16(26.6 \%)$ were free of a psychiatric diagnosis, 24 (40.0\%) met criteria for a single condition, and 20 (33.4\%) had two or more conditions.

Weight loss patterns also varied among participants, especially after the first year. For example, only a single laparoscopic adjustable gastric-banding patient and no gastric bypass patient failed to lose weight during the first year following surgery. However, a full $47.5 \%$ of participants either failed to lose any further weight after 1 year 
Table 1 Demographic, biomedical, and psychiatric descriptors of veteran sample $(N=60)$

\begin{tabular}{|c|c|c|}
\hline Variable & $\begin{array}{l}\text { Gastric } \\
\text { bypass } \\
(n=52)\end{array}$ & $\begin{array}{l}\text { Laparoscopic } \\
\text { adjustable } \\
\text { gastric banding } \\
(n=8)\end{array}$ \\
\hline Age, mean (SD) & $51.7(7.0)$ & $52.4(11.6)$ \\
\hline BMI at surgery* & $46.0(7.5)$ & $39.5(3.9)$ \\
\hline BMI at 6 months & $34.1(5.8)$ & $36.6(4.4)$ \\
\hline BMI at 12 months & $31.5(5.1)$ & $35.0(5.3)$ \\
\hline $\begin{array}{l}\text { Weight loss at } \\
12 \text { months* }\end{array}$ & $96.3(39.3)$ & $26.4(21.7)$ \\
\hline Percent weight loss at 12 -months* & 29.3 & 11.1 \\
\hline Percent female & 24.6 & 50 \\
\hline Percent non-Caucasian* & 23.1 & 58.3 \\
\hline Percent married & 43.1 & 50 \\
\hline Percent diabetic & 55.4 & 58.3 \\
\hline Percent hypertensive & 77.0 & 58.3 \\
\hline Percent dyslipidemia & 61.5 & 66.7 \\
\hline Percent sleep apnea & 58.5 & 66.7 \\
\hline $\begin{array}{l}\text { Percent erectile } \\
\text { dysfunction }\end{array}$ & 17.0 & 8.3 \\
\hline Percent depressed & 50.1 & 41.7 \\
\hline Percent anxiety disorder & 32.3 & 25.0 \\
\hline Percent binge eating & 27.8 & 8.3 \\
\hline $\begin{array}{l}\text { Percent substance } \\
\text { abuse history }\end{array}$ & 24.6 & 16.7 \\
\hline Percent smoking history & 30.8 & 33.3 \\
\hline Percent suicide attempt & 18.5 & 16.7 \\
\hline $\begin{array}{l}\text { Percent using } \\
\text { antidepressants }\end{array}$ & 47.7 & 33.3 \\
\hline Percent using anxiolytics & 18.5 & 0.0 \\
\hline
\end{tabular}

${ }^{*} p<0.05$, procedure groups differ

(18.0\%; 11 participants) or actually experienced weight regain between the 12- and 24-month measurement points (29.5\%; 18 participants).

Comparing 1-year weight loss percentages between participants grouped separately by depression (e.g., depression versus no depression), anxiety disorders, binge eating, and suicide attempt histories provided no support for the hypothesis that those with either of these diagnoses would lose less weight than those without the condition (all $p$ 's $>$ $0.10)$. Examining 1-year percent weight loss in relation to the number of psychiatric factors also failed to yield evidence of an association $(r=0.15, p>0.10)$. Among other demographic and biomedical factors, only dyslipidemia status $(r=-0.33, p=0.01)$ was associated with percent weight loss at 1 year.

Statistical relationships between psychiatric factors and weight loss improved when examining weight loss patterns after the first year. The percent BMI changes documented from 1 year post-surgery to 24 months post-surgery were significantly and inversely correlated with the participant's number of psychiatric conditions $(r=-0.30, p=0.02)$. Post hoc analyses completed using Tukey's honestly significant difference test indicated that participants with either one or two or more psychiatric conditions experienced significantly smaller percent of BMI changes $[1.5 \%,-3.9 \%$, and $-4.5 \%$ for those with zero, one, and two or more psychiatric conditions, respectively; $F(2,58)=3.6, p=0.03$ ] over the post-1-year follow-up than those with no psychiatric conditions.

As illustrated in Fig. 1, rates of weight loss cessation and weight regain increased in a dose-response pattern in relation to increasing numbers of psychiatric diagnoses. After adjusting for age, gender, race, type of weight loss procedure, and BMI at the time of surgery, patients with two or more psychiatric conditions were approximately six times more likely to either lose no additional weight from the 1-year post surgery or regain weight in comparison to those with no psychiatric diagnoses $(\mathrm{OR}=6.4,95 \% \mathrm{CI}=$ 1.3-12.4). After covariate adjustment, there was no difference between those with zero versus a single psychiatric diagnosis in terms of weight loss cessation/ weight regain $(\mathrm{OR}=1.01,95 \% \mathrm{CI}=0.25-4.1)$.

\section{Discussion}

This paper described associations between psychiatric factors and post-surgical outcomes in a veteran population completing either gastric bypass surgery or laparoscopic adjustable gastric banding. Consistent with the many prior studies in this area [9-13], we observed an absence of statistical relationships between psychiatric disorders and short-term weight loss defined by 1-year changes in weight and BMI. However, the major methodological shifts of the current study - emphasizing total psychiatric health over the presence of individual mental disorders and examining weight loss patterns after the first year - provided evidence of predictive value by suggesting a graded relationship between patient's number of psychiatric conditions and rates of weight regain or lack of further weight loss after 1 year post-surgery. The latter finding is not unprecedented, as a similar approach to aggregating mental health conditions was found to predict weight loss outcomes in a sample of 140 women completing a gastric banding procedure over a median follow-up of 50 months [17]. Despite the numerous differences in study population and methodology between the latter study and the current paper, this combination of findings suggests that a statistical approach using patient's complete mental health picture as opposed to the presence or absence of individual psychiatric diagnoses may have value in predicting weight loss surgery outcomes. 
Fig. 1 The relationship between patient's number of psychiatric factors and rates of weight loss cessation or weight regain 12 months following weight loss surgery

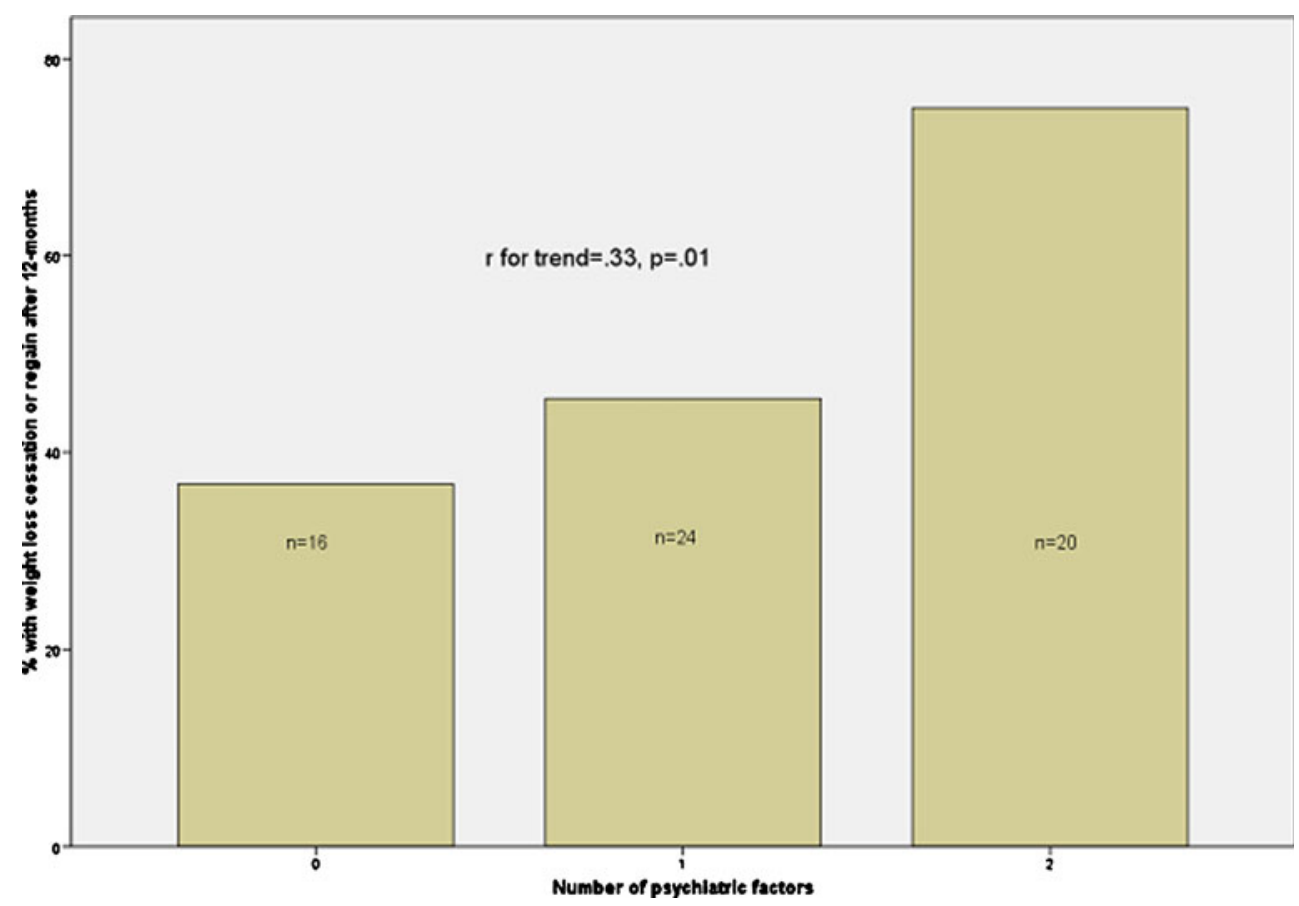

Despite the lack of strong evidence, to date, linking pre-surgical mental health status and weight loss following surgery, the prevalence and perceived importance of psychological evaluations in pre-weight loss surgery candidates remain high $[4,21,22]$. For example, in a survey study of 103 psychologists with experience completing pre-weight loss surgery evaluations, Walfish and colleagues [22] estimated that $15 \%$ of candidate patients are either delayed or denied due to psychological factors. Psychological factors are suspected to interfere with successful weight loss outcomes by undermining motivation, diet and exercise compliance, and other health behaviors critical to lasting weight loss [23]. Although the majority of patients affected by the results of pre-surgery psychological evaluations are delayed - usually referred to services to address identified concerns-rather than denied surgery, the volume of patients influenced by this practice can create interpretation difficulties in existing psychological research on post-bariatric outcomes. If the patients with the most substantial mental health problems are screened out or delayed until their concerns are amended as a result of the evaluation process, then the ability to find statistical relationships between psychological factors and weight loss surgery outcomes is sharply reduced through the reduction in variability in psychological status. The resulting disparity, wherein pre-surgical psychological evaluations are widely valued in practice and affect the surgical course of nearly one in six patients but whose benefits are difficult to confirm in much of the research to date, is an ongoing challenge for this area of study.
This study attempted to make a methodological compromise to this dilemma by examining each patient's total psychiatric status picture rather than the usual focus on single diagnoses in patients who had otherwise cleared their mental health evaluation. Our ability to make use of participant's computerized medical record may have been advantageous in these regards, as our psychiatric data were drawn not only from the standard pre-surgery psychological interview but also all other medical and mental health visits occurring the year of surgery. We also explored weight regain as a primary outcome measure, as a number of studies suggest that this complication affects $20 \%$ or more of weight loss surgery patients [18, 19, 24, 25]. In the current population, trends toward weight regain began early, with nearly $30 \%$ showing at least small weight gain after 1 year. Some studies (e.g., [25]) have reported that weight regain rates are higher among those with increased BMI; however, our study was not powered with enough severely obese patients to address this question. Instead, we found a relatively graded relationship between patients' number of psychiatric disorders and rates of weight loss cessation or weight regain after 1 year. This finding provides additional support for an aggregate approach for quantifying mental health status also used successfully by at least one other research group [17] but clearly requires replication in larger and more diverse samples.

Our second methodological divergence was to specifically examine psychiatric predictors of weight changes after the first year following surgery. Weight loss maintenancewhether following a lifestyle or surgical intervention - is a topic of increased focus in weight loss research (e.g., [26- 
28]). Although maximum weight loss is typically achieved in the first 1-2 years following weight loss surgery, longterm reports suggest that weight regain is troublingly common across a range of surgical treatments (e.g., [19, 29]). As a result, psychiatric data may become more important when examined in relation to weight changes after the early period of primarily surgery-induced weight loss, after which behavioral factors might emerge as primary factors in continued weight changes. If true, an emphasis in behavioral weight loss research on long-term outcomes is indicated. This hypothesis is consistent with our observed results, wherein the presence of one or more psychiatric diagnoses was associated with BMI changes documented after 1 year. In contrast, psychiatric factorweight relationships in the context of 1-year changes were, similar to many previous studies [9, 12, 13], not significant.

This study contains several design characteristics that place limits upon the findings. The participating sample was comprised exclusively of veterans, a population that is comparatively older, more likely to be male, and presenting with greater rates of both medical and psychiatric comorbidities compared to civilian groups [30,31]. Notably, this study also focused solely upon veterans using the VA healthcare system for their medical care, whereas some studies indicate that upward $75 \%$ of veterans do not use the VA [31]. For this reason, the results from this study cannot be widely applied to US veterans without appreciating that there are likely important differences between those who do and do not utilize the VA system for medical care.

The methods in which we derived medical status and psychiatric diagnoses can also be questioned. For example, participants in this study did not complete a structured psychiatric interview such as the Structured Clinical Interview for the DSM (SCID [5]), which limits our ability to infer psychiatric diagnoses specifically at the time of weight loss surgery. Instead, our identification of mental disorders was derived from several sources-including a pre-surgery unstructured interview with a licensed psychologist, charted psychiatric diagnoses, prescribed psychotropic medications at the time of surgery admission, and other concurrent mental health treatments. These methods may have contributed to the relatively high rates of single and multiple psychiatric disorders we observed in the sample, although it should be noted that our observed rate of $73.3 \%$ of patients reporting at least one of the measured psychiatric conditions is only marginally higher than the $66.3 \%$ rate of lifetime axis I disorder reported in a recent study using standardized clinical interviews [5]. Similarly, followup visits with patients were not structured but rather consisted of their routine clinical care following surgery. The latter created some minor inconsistency in follow-up dates and weights/BMI measure resulting from the use of different hospital scales.

\section{Summary}

In this study of veterans completing weight loss surgery, we observed a significant association between patient's number of psychiatric conditions and weight regain or the absence of further weight loss after 12 months following surgery. This relationship was strongest among those with two or more psychiatric conditions. Consistent with the majority of previous research, we did not identify significant relationships between psychiatric factors and short-term weight loss measured over the first year but did find that poor psychiatric health predicted less weight loss after the initial year. Given that pre-bariatric psychological evaluations likely already screen out those least suited to weight loss surgery success, patients that actually complete weight loss surgery are likely a population with reasonably intact mental health. This may account for the generally weak relationships reported in studies between psychological factors and weight loss and support the use of mental health variables either in combination or in relation to alternative clinical outcome measures such as weight regain in order to identify patients at risk for poor surgical results.

Open Access This article is distributed under the terms of the Creative Commons Attribution Noncommercial License which permits any noncommercial use, distribution, and reproduction in any medium, provided the original author(s) and source are credited.

\section{References}

1. Santry HP, Gillen DL, Lauderdale DS. Trends in bariatric surgical procedures. JAMA. 2005;294:1909-17.

2. Encinosa WE, Bernard DM, Du D, et al. Recent improvements in bariatric surgery outcomes. Med Care. 2009;47:531-5.

3. National Institute of Health Consensus Development Conference. Consensus Statement. Gastrointestinal surgery for severe obesity. March 25-27, 1991;9:1.

4. Bauchowitz AU, Gonder-Frederick LA, Olbrisch ME, et al. Psychosocial evaluation of bariatric surgery candidates: a survey of present practices. Psychosom Med. 2005;67(5):825-32.

5. Kalarchian MA, Marcus MD, Levine MD, et al. Psychiatric disorders among bariatric surgery candidates: relationship to obesity and functional health status. Am J Psychiatry. 2007;164:328-34.

6. Dziurowicz-Kozlowska AH, Wierzbicki Z, Lisik W, et al. The objective of psychological evaluation in the process of qualifying candidates for bariatric surgery. Obes Surg. 2006;16(2):196-202.

7. Grothe KB, Dubbert PM, O'jile JR. Psychological assessment and management of the weight loss surgery patient. Am J Med Sci. 2006;331(4):201-6.

8. Mechanick JI, Kushner RF, Sugerman HJ, et al. American Association of Clinical Endocrinologists, The Obesity Society, and American Society for Metabolic \& Bariatric Surgery Medical Guidelines for Clinical Practice for the perioperative nutritional, 
metabolic, and nonsurgical support of the bariatric surgery patient. Surg Obes Relat Dis. 2008;4(5 Suppl):S109-84.

9. van Hout GC, Verschure SK, van Heck GL. Psychosocial predictors of success following bariatric surgery. Obes Surg. 2005;15(4):552-60.

10. Herpertz S, Kielmann R, Wolf AM, et al. Do psychosocial variables predict weight loss or mental health after obesity surgery? A systematic review. Obes Res. 2004;12(10):1554-69.

11. Greenberg I, Perna F, Kaplan M, et al. Behavioral and psychological factors in the assessment and treatment of obesity surgery patients. Obes Res. 2005;13(2):244-9.

12. Bocchieri LE, Meana M, Fisher BL. A review of psychosocial outcomes of surgery for morbid obesity. J Psychosom Res. 2002;52:155-65.

13. Sarwer DB, Wadden TA, Fabricatore AN. Psychosocial and behavioral aspects of bariatric surgery. Obes Res. 2005;13:639-48.

14. van Hout GC, Hagendoren CA, Verschure SK, et al. Psychosocial predictors of success after vertical banded gastroplasty. Obes Surg. 2008;19:701-7.

15. Herpertz S, Kielman R, Wolf AM, et al. Does obesity surgery improve psychosocial functioning? A systematic review. Int $\mathrm{J}$ Obes. 2003;27:1300-14.

16. Bocchieri-Ricciardi LE, Chen EY, Munoz D, et al. Pre-surgery binge eating status: effect on eating behavior and weight outcome after gastric bypass. Obes Surg. 2006;16(9):1198-204.

17. Kinzl JF, Schrattenecker M, Traweger C, et al. Psychosocial predictors of weight loss after bariatric surgery. Obes Surg. 2006;16(12):1609-14.

18. Christou NV, Look D, Maclean LD. Weight gain after short- and long-limb gastric bypass in patients followed for longer than 10 years. Ann Surg. 2006;244(5):734-40.

19. Karlsson J, Taft C, Rydén A, et al. Ten-year trends in health-related quality of life after surgical and conventional treatment for severe obesity: the SOS intervention study. Int J Obes. 2007;31:1248-61.
20. Kaplan MS, Huguet N, McFarland BH, et al. Suicide among male veterans: a prospective population-based study. J Epidemiol Community Health. 2007;61:619-24.

21. Glinski J, Wetzler S, Goodman E. The psychology of gastric bypass surgery. Obes Surg. 2001;11:581-8.

22. Walfish S, Vance D, Fabricatore AN. Psychological evaluation of bariatric surgery applicants: procedures and reasons for delay or denial of surgery. Obes Surg. 2007;17(12):1578-83.

23. Sarwer DB, Wadden TA, Moore RH, et al. Preoperative eating behavior, postoperative dietary adherence, and weight loss after gastric bypass surgery. Surg Obes Relat Dis. 2008;4(5):640-6.

24. Meguid MM, Glade MJ, Middleton FA. Weight regain after Rouxen-Y: a significant $20 \%$ complication related to PYY. Nutrition. 2008;24(9):832-42.

25. Magro DO, Geloneze B, Delfini R, et al. Long-term weight regain after gastric bypass: a 5-year prospective study. Obes Surg. 2008;18(6):648-51.

26. Wing RR, Tate DF, Gorin AA, et al. A self-regulation program for maintenance of weight loss. N Engl J Med. 2006;355(15): 1563-71.

27. Marinilli Pinto A, Gorin AA, Raynor HA, et al. Successful weight-loss maintenance in relation to method of weight loss. Obesity (Silver Spring). 2008;16(11):2456-61.

28. Wing RR, Hill JO. Successful weight loss maintenance. Annu Rev Nutr. 2001;21:323-41.

29. Gracia JA, Martínez M, Elia M, et al. Obesity surgery results depending on technique performed: long-term outcome. Obes Surg. 2009;19:432-8.

30. Nelson KM. The burden of obesity among a national probability sample of veterans. J Gen Intern Med. 2006;21(9):915-9.

31. Kaplan MS, Huguet N, McFarland BH, Newsom JT. Suicide among male veterans: a prospective population-based study. J Epidemiol Community Health. 2007;61(7):619-24. Erratum in: J Epidemiol Community Health. 2007 Aug;61(8):751. 